\title{
Post-Stroke Cognitive Disorders and Associated Factors in French Speaking West Africa, Benin Case
}

\author{
Dieu Donné Gnonlonfoun1, Paul Macaire Ossou-Nguiet², Lansana Laho Diallo3, \\ Constant Adjien'1, Isaac Avlessi' ${ }^{1}$, Gérard Goudjinou1, Octave Houannou' ${ }^{1}$, \\ Dismand Houinato1, Gilbert Dossou Avode1 \\ ${ }^{1}$ Neurology Department, Centre National Hospitalier et Universitaire Hubert Koutoukou MAGA de Cotonou \\ (CNHU-HKM), Cotonou, Benin \\ ${ }^{2}$ Neurology Department, Centre Hospitalier et Universitaire of Brazzaville, Brazzaville, Congo \\ ${ }^{3}$ Neurology Department, Centre Hospitalier et Universitaire de Conakry, Conakry, Guinée \\ Email: dignon2002@yahoo.fr
}

Received 2 January 2014; revised 1 February 2014; accepted 22 February 2014

Copyright (C) 2014 by authors and Scientific Research Publishing Inc.

This work is licensed under the Creative Commons Attribution International License (CC BY).

http://creativecommons.org/licenses/by/4.0/

(c) (i) Open Access

\section{Abstract}

Introduction: Cognitive disorders frequency arising after a cardio-cerebral vascular disease (stroke) is currently on the rise due to the ageing population and the increase in the number of survivors after stroke occurrence. Objective: Determining post-stroke cognitive decline and identifying associated factors. Method: It consists in a cross-sectional, prospective, descriptive and analytical study which was conducted from April 1 to August 31, 2013 in the Neurology Department of CNHU-HKM in Cotonou. The study involved 100 patients who have known stroke for at least the past 6 months and were all admitted and discharged later on. The disease survivors were re-contacted and examined again at home or at hospital. The cognitive decline (CD) was estimated by using a modified and adapted MMSE to suit our cultural era. Results: All patients were aged 58.9 years \pm 13.6 years. Sex-ratio was 1.4 . Cognitive decline frequency was $20 \%$. Post-stroke cognitive decline frequency per sex was $11.6 \%$ and $8.4 \%$ respectively for females and males. Ischemia stroke patients had a higher cognitive decline $(22.5 \%) .58 .3 \%$ had severe CD. Moreover the CD frequency increased with time, from $16.7 \%$ in a year to $50 \%$ in 3 years. Total MMSE varied from 6 to 23 with 21 as median. From the unvaried analysis, the presence of sphincter disorders (1.26 [0.35 - 4.59], $p=0.004)$, consciousness disorders $(15.67[1.46-168], p=0.04)$, and convulsion disorders (3.77 $[1.01-14.00], p=0.003)$ was associated with cognitive decline. From multi-varied analysis, the sole presence of convulsive attacks $(11.5[1.79-73.58], p=0.01)$ was individually associated with post-stroke cognitive decline. Conclusion: This study reveals the importance of $C D$ after stroke. The occurrence of convulsions at stroke acute stage is a prognostic factor of CD medium or long-term occurrence. The overall coverage improvement depends on the integration of these data in stroke reach-out programs. 


\section{Keywords}

\section{Stroke; Cognitive Decline; Dementia; Benin}

\section{Introduction}

According to the World Health Organization, stroke is a sudden neurological deficit of vascular origin. It is a serious pathology with a serious impact on the functional and vital prognosis [1]. Throughout the world, stroke represents the second cause of dementia behind Alzheimer's disease [2]. With a frequency of $44.4 \%$, stroke constitutes in Benin the most frequent malady subject of hospitalization in the Neurology Department of CNHU-HKM in Cotonou [3]. It has a serious impact on patients' social life, considering post-stroke days full of sufferings in the form of physical, emotional and cognitive symptoms which are really distressing [4]. The relationship between initial deficiencies and subsequent recovery is greatly variable, and this makes it difficult to conduct exact prognosis for each patient [5]. Several studies were initiated and conducted on stroke in Benin hospitals. In view of knowing the disease better in order to provide patients with effective coverage following their discharge from hospital, this study was initiated and its main objective is determining the prevalence of post-stroke cognitive decline and identifying associated factors.

\section{Method}

It consists in a cross sectional, prospective, descriptive and analytical study which was conducted from April 1, 2013 to August 31, 2013 in Hubert Koutoukou Maga teaching hospital of Cotonou Bénin. Benin is a French Speaking country in West African (Figure 1) sharing border with Nigeria on the east. It boasts of 9 million inhabitants and an area of $112.622 \mathrm{~km}^{2}$. The population subjected to study consisted of patients suffering from stroke at least for the past 6 months. The sample size was calculated using Daniel Schwartz formulae $\mathrm{n}=\mathrm{Z} \alpha^{2}$ $\mathrm{pq} / \mathrm{i}^{2}=96$ with $\mathrm{p}=48.3 \%$ (stroke prevalence in the neurology department at CNHU-HKM [6], $\alpha=5 \%$ and I $=$ $10 \%)$. But the total number of subjects enrolled in the study period was 100. During the study period, we performed a systematic enrollment of all patients who met the inclusion criteria and consented to participate in the study, up to the number expected.

\subsection{Inclusion Criteria}

- Having suffered from stroke and been treated in the Department of Neurology;

- Having been suffering from stroke for at least 6 months;

- Having provided one's contacts in the medical record during hospitalization.

\subsection{Non-Inclusion Criteria}

- Any patient with a meningeal hemorrhage, cerebral venous thrombosis or a neurological deficit associated with head injury or brain tumor or other cause;

- Exception of brain scan.

\subsection{Exclusion Criteria}

Presence of aphasia during the study.

\subsection{Collection Modalities}

From the register-book and database made available by the data service, all patients having suffered from stroke and meeting the inclusion criteria were identified up to the sample size. Only those who survived stroke and were discharged after hospitalization were contacted via telephone. Those who were still alive were re-examined either at hospital or in their home on appointment basis. A clinical examination of the patient was then carried out together with a set of questionnaires. The data were supplemented by the analysis of patient medical records. 


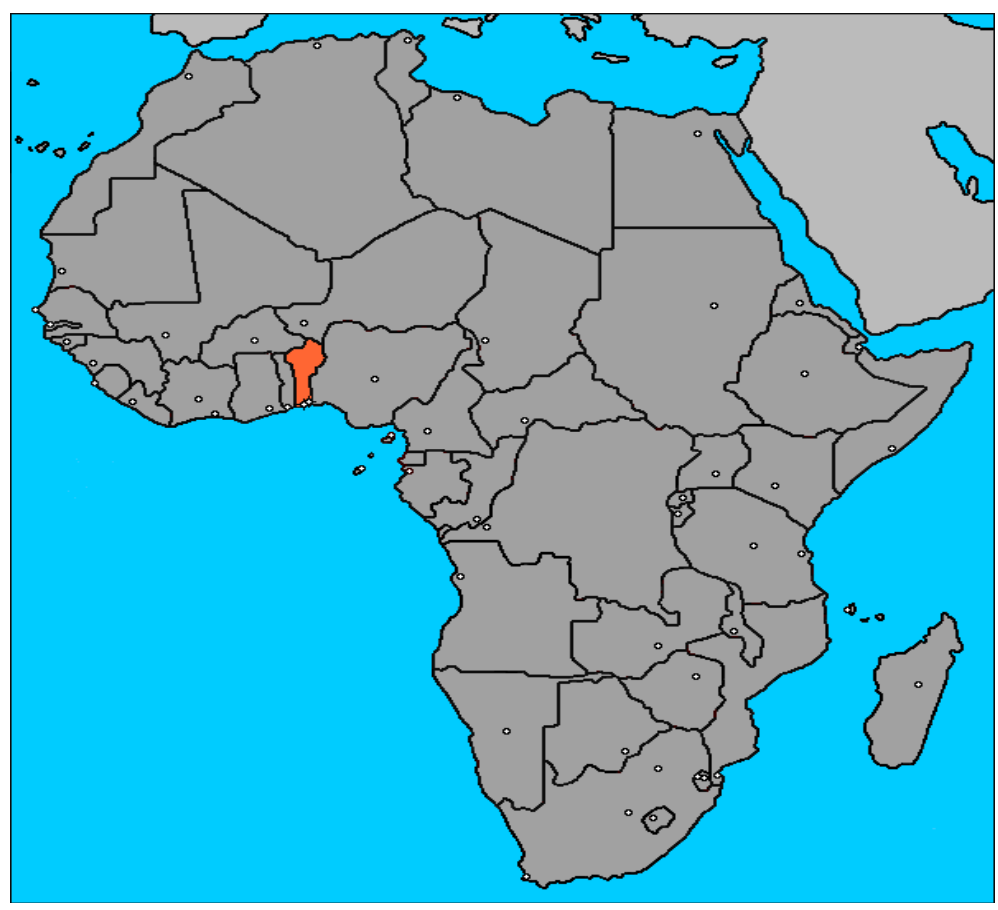

Figure 1. Position of Bénin in Africa.

\subsection{The Variables Studied Were}

Dependent variable: the cognitive decline which was estimated using Mini Mental State Examination (MMSE) modified and adapted to our cultural era. MMSE equal or below 24 was considered as cognitive decline.

- MMSE between 24/30 and 20/30 was considered as "moderate cognitive decline".

- MMSE below 19/30 was considered as "severe cognitive decline".

Independent variables:

- The socio-demographic data (age, gender, ethnicity, occupation, place of residence, marital status, laterality, education level, monthly income).

- Past record (hypertension, diabetes, hyperlipidemia, hormonal contraception, coronary past record, stroke, peripheral arterial disease of the lower limbs, vascular surgery, heart disease, dementia, and migraine).

- The patient's lifestyle (alcohol, tobacco, inactivity, others).

- Data available when stroke occurred: date of stroke occurrence, stroke type, stroke topography, hemi-corpus in deficit, hypertension, deficit type, aphasia, consciousness disorder, seizures, sphincter disorders, metabolic disorders, time-limit between stroke occurrence and the day the survey was conducted, the use of traditional medicine after hospitalization, duration of hospitalization, recurrence after hospitalization, physiotherapy and any other rehabilitation act (motor, speech therapy, occupational therapy).

- Current clinical data: blood pressure, BMI, mobility.

\subsection{Data Processing and Analysis}

Data processing was conducted through EPI-DATA. Audit and data analysis were done using statistics software STATA/IC 11.0. A descriptive analysis was completed with regard to the variables which were studied. So, as far as qualitative variables were concerned, frequencies and proportions were determined. Either chi2 or FISHER test was used if only expected values are lower than 5 . For quantitative values, averages together with their typical gaps, medians, minima and maxima have all been described. STUDENT test was utilized for comparisons. The study of associated factors was conducted using logistical regression model in unvaried and multivaried analyses. The multi-varied analysis was carried out by inserting into the model all variables, of which $p$ value in unvaried analysis is less or equal to $20 \%$ because of the exploratory nature of the study. The break-even point in terms of significance was $5 \%$ and confidence gap rated at $95 \%$. 


\subsection{Ethical Considerations}

Each patient or his/her next of kin submitted a written letter of consent upon explanation of the study objective and modalities.

\section{Results}

\subsection{Socio-Demographic Characteristics of the Population}

All in all 100 patients were enrolled. Their age varied between 18 to 86 years with an average of 58.9 years \pm 13.6 years. There were $58 \%$ of males against $42 \%$ of females meaning a sex-ratio $(\mathrm{M} / \mathrm{F})$ of 1.4 .

\subsection{Cognitive Decline Frequency}

MMSE was conducted on sixty patients (aphasia patients were excluded); 12 had a cognitive decline, meaning $20 \%$ frequency. The cognitive decline was more recurrent with stroke patients aged over 70 years (23.5\%) than other age categories $(\mathrm{p}=0.03$ ) as indicated in Figure 2. Post-stroke females cognitive decline frequency was $11.6 \%$ while it was $8.4 \%$ with males. $22.5 \%$ of ischemia stroke patients presented cognitive disorders as shown in Table 1. 58.3\% of patients presented a severe cognitive decline after stroke (Figure 3). Post-stroke cognitive decline frequency increased with time, from $16.7 \%$ in a year to 50\% in 3 years as indicated in Figure 4.

\subsection{Characteristic of the MMSE}

The total MMSE ranged from 6 to 23 with a median of 21. Table 2 shows the classification of different MMSE items with regard to subjects with cognitive decline. $50 \%$ of patients who had an ischemic stroke had a temporal disorientation against 25\% for hemorrhagic stroke subjects. Table 3 summarizes the classification of various cognitive impairment related to the MMSE observed in both ischemia stroke patients and hemorrhagic stroke.

\subsection{Factors Associated with Post-Stroke Cognitive Decline}

During the unvaried analysis, among the elements included in the socio-demographic data, only sex (IC $95 \%=2.8$ [1.77 to 3.22], $\mathrm{p}=0.041$ ) was found as associated with the cognitive decline after stroke, as shown in Table 4. However, no past record is significantly associated with cognitive decline (Table 5). Clinically, the presence of sphincter disorder ( $\mathrm{IC}_{95 \%}=1.26$ [0.35 to 4.59], $\left.\mathrm{p}=0.004\right)$, consciousness disorder $\left(\mathrm{IC}_{95 \%}=15.67\right.$ [1.46 - 168 ], $\mathrm{p}$ $=0.04$ ), and convulsion ( $\mathrm{IC}_{95 \%}=3.77$ [1.01 to 14.00], $\mathrm{p}=0.003$ ) at stroke occurrence, is associated with cognitive decline. Table 6 summarizes the data.

\subsection{Cognitive Decline Prognosis}

After multi-varied analysis and logistic regression, only the presence of convulsions ( $\mathrm{IC}_{95 \%}=11.5$ [1.79 to 73.58], $\mathrm{p}=0.01$ ) is individually associated with post-stroke cognitive decline, at stroke occurrence as shown in Table 7 .

\section{Discussion}

It is a cross-sectional and prospective study with descriptive and analytical aims. The method adopted met the requirements of the type of study conducted. The calculated sample size was 96 and 100 stroke patients were finally enrolled. But the limitation of this study is the small number of subjects with cognitive impairment.

The prevalence of post-stroke cognitive decline observed in our study is $20 \%$. This frequency is close to the 23\% found by Garcia et al. in 2013 [7]. However, higher frequencies have been observed by several authors. Thus, Odjagbemi et al., in a study of post-stroke cognitive impairment conducted in Nigeria in 2013, observed a frequency of 27.9\% [8]. At Dijon, in France, in 2012, Jacquin et al. [9], on his side reported a frequency of post-stroke cognitive disorders estimated at $45.5 \%$. This difference is due to the fact that, these two studies considered as post-stroke cognitive impairment an MMSE lower or equal to 26/30 whilst in ours, the presence of cognitive impairment was defined by an MMSE modified and adapted to our cultural area which is lower or equal to 24/30. All these results show that cognitive decline is quite common in the medium and long term with patients who suffered from stroke. 


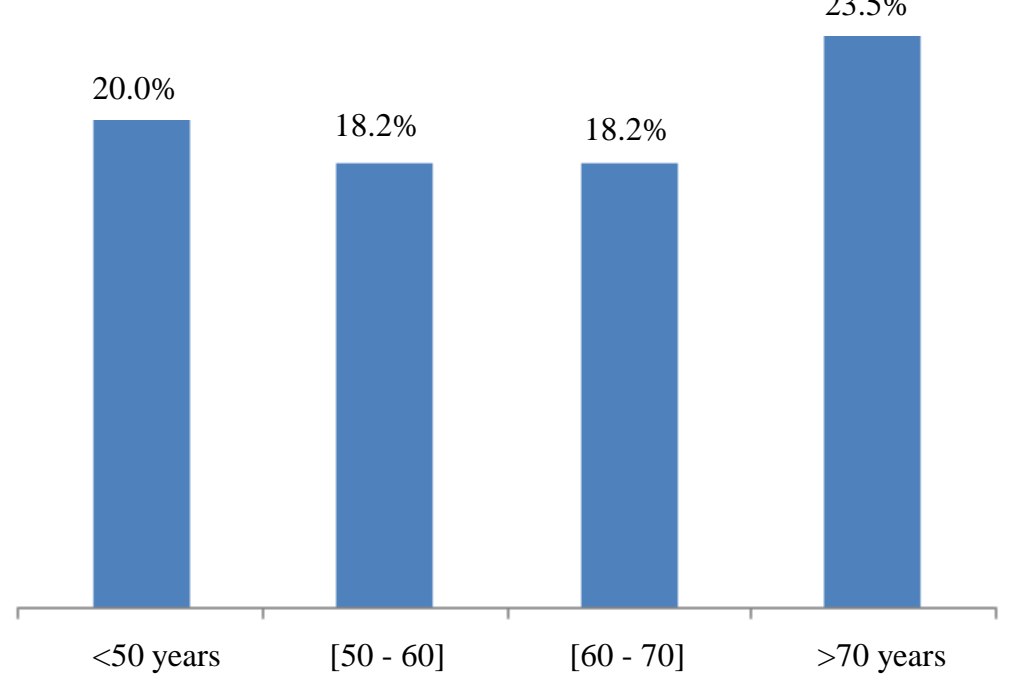

Figure 2. Classification of cognitive decline per age, Cotonou 2013.

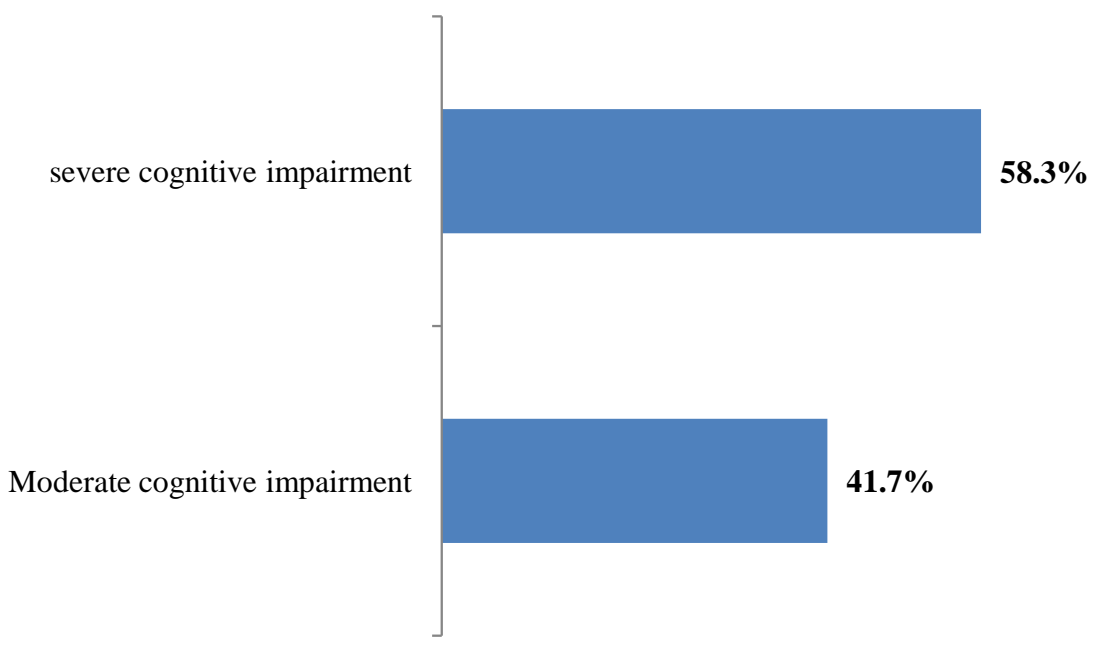

Figure 3. Classification of cognitive decline severity, Cotonou 2013.

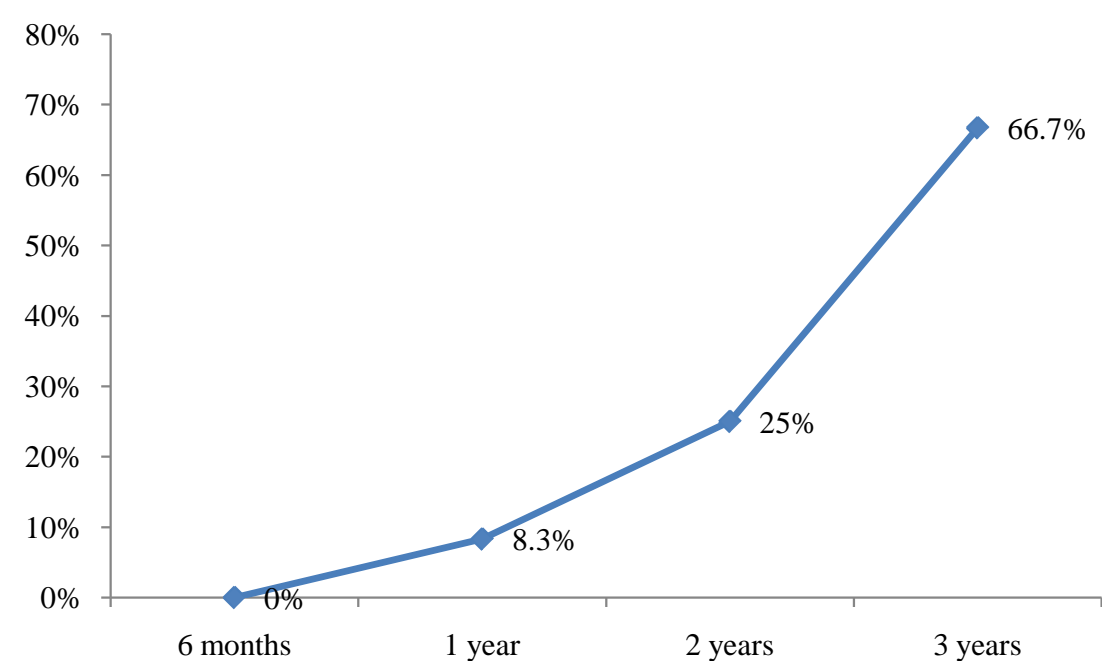

Figure 4. Cognitive decline frequency per time spent after stroke, Cotonou 2013. 
Table 1. Cognitive decline per stroke type, Cotonou 2013.

\begin{tabular}{cccc}
\hline Cognitive condition & Ischemic stroke $\mathbf{n}(\%)$ & Hemorrhagic stroke $\mathbf{n}(\%)$ & total \\
\hline No decline & $31(77.5)$ & $17(85.0)$ & 48 \\
Cognitive decline & $9(22.5)$ & $3(15.0)$ & 12 \\
Total & $\mathbf{4 0}$ & $\mathbf{2 0}$ & $\mathbf{6 0}$ \\
\hline
\end{tabular}

$\mathrm{p}=0.115$.

Table 2. Characteristics of MMSE score, Cotonou 2013.

\begin{tabular}{ccc}
\hline MMSE items & Median score [Mini - Max $]$ \\
\hline Time concept & $3[0-5]$ \\
Space concept & $4[0-5]$ & $2[2-3]$ \\
Training & $0[0-2]$ & $0[0-1]$ \\
Reflection and calculation & $6[2-8]$ & $1[0-1]$ \\
Memory capacity & Language & \\
\hline
\end{tabular}

Table 3. Cognitive disorders frequency with mad subjects, Cotonou 2013.

\begin{tabular}{ccc}
\hline Various disorders & Ischemic stroke n (\%) & Hemorrhagic stroke n (\%) \\
Concept & & $3(25 \%)$ \\
$-\quad$ Time & $6(50 \%)$ & $2(16.7 \%)$ \\
Training & $3(25 \%)$ & $2(16.7 \%)$ \\
Reflection and calculation & $6(50 \%)$ & $3(25 \%)$ \\
Memory capacity & $6(50 \%)$ & $3(25 \%)$ \\
Language & $3(25 \%)$ & $1(8.3 \%)$ \\
Constructional apraxia & $3(25 \%)$ & $1(8.3 \%)$ \\
\hline
\end{tabular}

Table 4. Socio-demographic factors associated with cognitive decline, Cotonou 2013.

\begin{tabular}{|c|c|c|c|c|}
\hline Socio-demographic variables & Total & Cognitive decline $\mathbf{n}(\%)$ & $\mathrm{RC}\left[\mathrm{IC}_{95 \%}\right]$ & Value p \\
\hline Age (years) & & & & 0.99 \\
\hline$<50$ & 17 & $4(23.5)$ & 1 & \\
\hline$[50-60]$ & 22 & $4(18.2)$ & $0.72[0.15-3.43]$ & \\
\hline$[60-70]$ & 11 & $2(18.2)$ & $0.72[0.11-4.82]$ & \\
\hline$>70$ & 10 & $2(20.0)$ & $0.81[0.12-5.50]$ & \\
\hline Gender & & & & $0.041^{*}$ \\
\hline Male & 37 & $5(13.5)$ & 1 & \\
\hline Female & 23 & $7(30.4)$ & $2.8[1.77-3.22]$ & \\
\hline Marital status & & & & 0.405 \\
\hline Single & 19 & $5(26.3)$ & 1 & \\
\hline Marital life & 41 & $7(17.1)$ & $0.58[0.15-2.13]$ & \\
\hline Life style & & & & 0.553 \\
\hline Alcohol & 31 & $5(16.1)$ & 1 & \\
\hline Tobacco & 3 & 1 (33.3) & $2.6[0.20-34.4]$ & \\
\hline Sedentary living & 18 & $5(27.8)$ & $2[0.49-8.17]$ & \\
\hline Others & 2 & $1(50.0)$ & $5.2[0.28-97.61]$ & \\
\hline Profession & & & & 0.375 \\
\hline Civil servant & 19 & $2(10.5)$ & 1 & \\
\hline Private sector employee & 8 & $1(12.5)$ & $1.21[0.94-15.65]$ & \\
\hline Craftsman & 9 & $3(33.3)$ & $4.25[0.57-31.65]$ & \\
\hline Housewife & 15 & $5(33.3)$ & $4.25[0.69-26.13]$ & \\
\hline Pensioner & 8 & $1(12.5)$ & $1.21[0.94-15.65]$ & \\
\hline
\end{tabular}

"Significant result. 
Table 5. Past medical records associated with cognitive decline, Cotonou 2013.

\begin{tabular}{ccccc}
\hline Past records & Total & Cognitive decline n (\%) & RC [IC $95 \%]$ & Value $\mathbf{p}$ \\
\hline $\begin{array}{c}\text { High Blood Pressure } \\
\text { No hypertension } \\
\text { Hypertension }\end{array}$ & 10 & $2(20.0)$ & 1 & 0.507 \\
Diabetes & 50 & $10(20.0)$ & $1[0.16-6.45]$ & 0.634 \\
No diabetes & 55 & $11(20.0)$ & 1 & 0.686 \\
Diabetes & 5 & $1(20.0)$ & $2.14[0.10-4.86]$ & 1 \\
Cardiopathy & & & & $1(20.0)$ \\
No cardiopathy & 55 & $1(20.0)$ & $10.10-9.86]$ \\
Cardiopathy & 5 & & \\
\hline
\end{tabular}

Table 6. Clinical factors associated with cognitive decline, Cotonou 2013.

\begin{tabular}{|c|c|c|c|c|}
\hline Factors & Total & Motor deficit n (\%) & RC $\left[\right.$ IC $\left._{95 \%}\right]$ & Value $p$ \\
\hline Handicapped side & & & & 0.725 \\
\hline Right & 37 & $7(18.9)$ & 1 & \\
\hline Left & 22 & $5(22.7)$ & $1.26[0.35-4.59]$ & \\
\hline Sphincter disorders & & & & $0.004^{*}$ \\
\hline No & 56 & $9(16.1)$ & 1 & \\
\hline Yes & 4 & $3(75.0)$ & $15.67[1.46-168]$ & \\
\hline Consciousness disorders & & & & $0.040^{*}$ \\
\hline No & 40 & $5(12.5)$ & 1 & \\
\hline Yes & 20 & $7(35.0)$ & $3.77[1.01-14.00]$ & \\
\hline Convulsion & & & & $0.003^{*}$ \\
\hline No & 54 & $8(14.8)$ & 1 & \\
\hline Yes & 6 & $4(66.7)$ & 11.5 [1.80 - 73.57] & \\
\hline Metabolic disorders & & & & 0.744 \\
\hline No & 49 & $9(18.4)$ & 1 & \\
\hline Yes & 4 & $1(25.0)$ & $1.48[0.14-15.94]$ & \\
\hline
\end{tabular}

*Significant result.

Table 7. Post-stroke cognitive decline prognosis, Cotonou 2013.

\begin{tabular}{cccccc}
\hline Variables & Total & Decline $\mathbf{n}(\%)$ & Gross RC & Adjusted RC & Value p \\
\hline Convulsion & & & & & \\
No & 54 & $8(14.8)$ & 1 & 1 & $11.5[1.79-73.58]$ \\
Yes & 6 & $4(66.7)$ & $11.5[1.80-73.57]$ & $0.010^{*}$ \\
\hline
\end{tabular}

${ }^{*}$ Significant result.

We noted an increase in the frequency of cognitive decline with age. Indeed, 20\% of patients below 50 years had risk factor cognitive decline but beyond 70 years, the frequency reached $23.5 \%$. The greatest known cognitive decline risk factor and dementia is age. Most people with the disease are 65 years or above. Individuals aged 30 to 40 years may experience early onset of Alzheimer's disease if there is a family past medical record related to the condition. The probability of developing Alzheimer's disease every five years after 65 years old is doubled. After 85 years old, the risk reaches nearly 50\% [10]. Debette, in his review of the literature on "Vascular Risk Factors and cognitive disorders” in 2013 [11], states that the prevalence of cognitive impairment increases as we get older. Age could double the risk of developing cognitive impairment $\left(\mathrm{HR}=1.9\right.$ [ $\mathrm{IC}_{95 \%}=1.3-$ 2.8]). When one gets older, there is a process of resistance decline and the effectiveness of the body in addition 
to slowdown in physiological function. This condition leads to cerebral degenerative lesions leading to cognitive impairment [12]-[14].

The classification of cognitive decline per gender shows a female predominance with $11.6 \%$ against $8.4 \%$ for male. Most studies have confirmed an increase in the incidence and prevalence of dementia in women females [4] [15]. Indeed, in our studied population, in Benin, the level of education of males, in most cases, is higher than that of female. Thus, the majority of females included in the study were housewives or retailers. Knowing that the level of education has an influence on the MMSE, one could understand this female predominance.

The frequency of the post ischemia stroke cognitive decline (22.5\%) is higher than the post hemorrhagic stroke cognitive decline (15.0\%). Ischemia stroke is then a lot more involved in the occurrence of long-term cognitive decline than hemorrhagic stroke. Hénon et al. [5], in Lille in France made the same observation in 2001 with $23 \%$ of cognitive decline per ischemia stroke against $10 \%$ per hemorrhagic stroke. The occurrence of post-stroke dementia is also more common in patients with silent cerebral infarctions [2]. Silent cerebral infarctions are associated with mild cognitive impairment [16]. Cordonnier et al., in their review of literature on "subclinical strokes and dementia" in 2011, showed that the mechanism by which silent cerebral infarcts and cognitive decline are linked is not fully elucidated. An amplification effect of vascular and possibly degenerative lesions, resulting in a lesion load that is enough to cause cognitive decline is discussed [17]. It is also possible that these lesions promote the development of amyloid plaques and neurofibrillary degeneration [18]. Clinic-pathological studies have shown that the probability to have dementia is higher with regard to subjects with lacunar infarctions and this requires less amyloid plaques and neurofibrillary degeneration for an equivalent level of cognitive decline [19]. Beside the lesion volume, infarction location also plays a role in the occurrence of cognitive impairment: thalamic locations, especially in frontal and dorso-median nucleus, are followed by short-term encoding and memory disorders, and promote the development of cognitive decline [17]. After multi-varied statistical analysis and logistic regression, the long-term cognitive decline prognosis found in this study is the occurrence of convulsions during stroke incident. To our knowledge, this association has not been described in the literature. However, a number of hypotheses can be put up to explain such association. Indeed, at the acute stage of infarction, the secondary hypoxia tissue dipping in terms of cerebral blood flow, the release of excitotoxic amino acids such as glutamate and the intracellular accumulation of calcium and sodium resulting in neuronal depolarization might be the cause of attacks [20]-[22]. Repetitive neuronal release may occur within networks of surviving neurons exposed to glutamate. A decrease in the efficiency of GABAergic inhibition resulting in a cortical excitability through NMDA receivers can also be mentioned. The ischemic penumbra zone could also play a role in the onset of attacks. The low frequency of early attacks suggests that factors other than ischemia play a triggering role: local factors (reperfusion lesions through early repermeabilization) or general factors (hyperglycemia, detoxification, electrolyte disorders, acid-base, pneumonia secondary to hypoxia...). The respective role of mass effect, associated ischemia, and hemoglobin degradation products is discussed in the genesis of hemorrhagic stroke secondary attacks [23]. Strokes provoke convulsions. These convulsive attacks, in turn, lead to milder brain damage that eventually could be the cause of cognitive decline.

\section{Conclusion}

This study reveals the importance of CD after stroke. The occurrence of convulsions at stroke acute stage is a prognostic factor of CD medium or long-term occurrence. The overall coverage improvement depends on the integration of these data in stroke reach-out programs.

\section{References}

[1] Sène-Diouf, F., Basse, M., Ndao, K. and Ndiaye, M. (2006) Functional Prognosis of Stroke in Developing Countries: Senegal. Annales de Readaptation et de Medecine Physique, 49, 100-104.

[2] Leys, D., Henon, H., Mackowiak-Cordoliani, M.A. and Pasquier, F. (2005) Poststroke Dementia. Lancet Neurology, 4, 752-759. http://dx.doi.org/10.1016/S1474-4422(05)70221-0

[3] Gnonlonfoun, D., Adjien, K.C., Ossou-Nguiet, P.M., Mapoure, Y., Houinato, D. and Avode, D.G. (2013) Hospitalization Frequency of Neurology Diseases at CNHU-HKM in Cotonou. Journal of the Clinical Biology Society, 18, 43-48.

[4] Nitrini, R., Caramelli, P., Herrera, E.J., Bahia, V.S., Caixeta, L.F., Radanovic, M., Anghinah, R., Charchat-Fichman, H., Porto, C.S., Carthery, M.T., Hartmann, A.P., Huang, N., Smid, J., Lima, E.P., Takada, L.T. and Takahashi, D.Y. (2004) Incidence of Dementia in a Community-Dwelling Brazilian Population. Alzheimer Disease and Associated 
Disorders, 18, 241-246.

[5] Hénon, H., Durieu, I., Guerouaou, D., Lebert, F., Pasquier, F. and Leys, D. (2001) Poststroke Dementia Incidence and Relationship to Prestroke Cognitive Decline. Neurology, 57, 1216-1222. http://dx.doi.org/10.1212/WNL.57.7.1216

[6] Houinato, D., Avode, D.G., Adjien, C. and Kindjinou, M. (2005) Epidemiological Profile of Stroke in Cotonou Hospitals. The Medical Benin, 29, 54-56.

[7] Garcia, P.Y. and Roussel, M. (2013) Cognitive Impairment and Dementia after Intracerebral Hemorrhage: A CrossSectional Study of a Hospital-Based Series. Journal of Stroke and Cerebrovascular Diseases, 22, 80-86. http://dx.doi.org/10.1016/j.jstrokecerebrovasdis.2011.06.013

[8] Ojagbemi, A. and Owolabi, M. (2013) Predictors of Functional Dependency after Stroke in Nigeria. Journal of Stroke and Cerebrovascular Diseases, 22, 381-387. http://dx.doi.org/10.1016/j.jstrokecerebrovasdis.2013.04.015

[9] Jacquin, A. (2013) Clinic and Scanning Factors Associated with Post Stroke Cognitive Disorders. Epidemiology and Public Health Review, 61, 82-85.

[10] Alonso, A., Mosley, J.T., Gottesman, R.F., Catellier, D., Sharrett, A.R. and Coresh, J. (2009) Risk of Dementia Hospitalisation Associated with Cardiovascular Risk Factors in Midlife and Older Age: The Atherosclerosis Risk in Communities (ARIC) Study. Journal of Neurology, Neurosurgery \& Psychiatry, 80, 1194-1201. http://dx.doi.org/10.1136/jnnp.2009.176818

[11] Debette, S. (2013) Vascular Risk Factors and Cognitive Disorders. Neurology Review, 169, 757-764. http://dx.doi.org/10.1016/j.neurol.2013.07.022

[12] Wen, W., Sachdev, P.S., Li, J.J., Chen, X. and Anstey, K.J. (2009) White Matter Hyperintensities in the Forties: Their Prevalence and Topography in an Epidemiological Sample Aged 44-48. Human Brain Mapping, 30, 1155-1167. http://dx.doi.org/10.1002/hbm.20586

[13] Debette, S., Beiser, A., Decarli, C., Au, R., Himali, J.J. and Kelly-Hayes, M. (2010) Association of MRI Markers of Vascular Brain Injury with Incident Stroke, Mild Cognitive Impairment, Dementia, and Mortality. The Framingham Offspring Study. Stroke, 41, 600-606. http://dx.doi.org/10.1161/STROKEAHA.109.570044

[14] Barnes, D.E. and Yaffe, K. (2011) The Projected Effect of Risk Factor Reduction on Alzheimer’s Disease Prevalence. Lancet Neurology, 10, 819-828. http://dx.doi.org/10.1016/S1474-4422(11)70072-2

[15] Letenneur, L., Gilleron, V., Commenges, D., Helmer, C., Orgogozo, J.M. and Dartigues, J.F. (1999) Are Sex and Educational Level Independent Predictors of Dementia and Alzheimer's Disease? Incidence Data from the PAQUID Project. Journal of Neurology, Neurosurgery \& Psychiatry, 66, 177-183. http://dx.doi.org/10.1136/jnnp.66.2.177

[16] Lopez, O.L., Jagust, W.J., Dulberg, C., Becker, J.T., DeKosky, S.T. and Fitzpatrick, A. (2003) Risk Factors for Mild Cognitive Impairment in the Cardiovascular Health Study Cognition Study: Part 2. Archives of Neurology, 60, 13941399. http://dx.doi.org/10.1001/archneur.60.10.1394

[17] Cordonnier, C. and Leys, D. (2011) “Silent” Vascular Lesions and Dementia. Neurology Practice-FMC, 2, $149-157$.

[18] Vermeer, S.E., Prins, N.D., den Heijer, T., Hofman, A., Koudstaal, P.J. and Breteler, M.M. (2003) Silent Brain Infarcts and the Risk of Dementia and Cognitive Decline. New England Journal of Medicine, 348, 1215-1222. http://dx.doi.org/10.1056/NEJMoa022066

[19] Jellinger, K.A. and Attems, J. (2003) Incidence of Cerebrovascular Lesions in Alzheimer's Disease: A Postmortem Study. Acta Neuropathologica (Berl), 105, 14-17.

[20] Silverman, I.E., Restrepo, L. and Mathews, G.C. (2002) Poststroke Seizures. Archives of Neurology, 59, $195-201$. http://dx.doi.org/10.1001/archneur.59.2.195

[21] Camilo, O. and Goldstein, L.B. (2004) Seizures and Epilepsy after Ischemic Stroke. Stroke, 35, 1769-1775. http://dx.doi.org/10.1161/01.STR.0000130989.17100.96

[22] Ferro, J.M. and Pinto, F. (2004) Poststroke Epilepsy: Epidemiology, Pathophysiology and Management. Drugs Aging, 21, 639-653. http://dx.doi.org/10.2165/00002512-200421100-00003

[23] Lamy, C. (2008) Epilepsy and Stroke. Neurology Review, 164, 841-845. http://dx.doi.org/10.1016/j.neurol.2008.07.006 


\section{Abbreviation List}

CHU: Centre Hospitalo-Universitaire

CD: Cognitive Decline

MMSE: Mini Mental State Examination

CNHU-HKM: Centre National Hospitalier et Universitaire Hubert Koutoukou Maga

GABA: Gamma-Aminobutyrique Acid

NMDA: N-Methyl-D-Aspartate 